\title{
Can MRI Findings Help to Predict Neurological Recovery in Paraplegics With Thoracolumbar Fracture?
}

\author{
Joonchul Lee, MD, Seong-Eun Koh, MD, Heeyoune Jung, MD, Hye Yeon Lee, MD, In-Sik Lee, MD \\ Department of Rehabilitation Medicine, Konkuk University School of Medicine and \\ Konkuk University Medical Center, Seoul, Korea
}

Objective To evaluate the usefulness of various magnetic resonance imaging (MRI) findings in the prognosis of neurological recovery in paraplegics with thoracolumbar fracture using association analysis with clinical outcomes and electrodiagnostic features.

Methods This retrospective study involved 30 patients treated for paraplegia following thoracolumbar fracture. On axial and sagittal T2-weighted MRI scans, nerve root sedimentation sign, root aggregation sign, and signal intensity changes in the conus medullaris were independently assessed by two raters. A positive sedimentation sign was defined as the absence of nerve root sedimentation. The root aggregation sign was defined as the presence of root aggregation in at least one axial MRI scan. Clinical outcomes including the American Spinal Injury Association impairment scale, ambulatory capacity, and electrodiagnostic features were used for association analysis.

Results Inter-rater reliability of the nerve root sedimentation sign and the root aggregation sign were $\kappa=0.67$ $(\mathrm{p}=0.001)$ and $\kappa=0.78(\mathrm{p}<0.001)$, respectively. A positive sedimentation sign was significantly associated with recovery of ambulatory capacity after a rehabilitation program $\left(\chi^{2}=4.854, \mathrm{p}=0.028\right)$. The presence of the root aggregation sign was associated with reduced compound muscle action potential amplitude of common peroneal and tibial nerves in nerve conduction studies $\left(\chi^{2}=5.026, \mathrm{p}=0.025\right)$.

Conclusion A positive sedimentation sign was significantly associated with recovery of ambulatory capacity and not indicative of persistent paralysis. The root aggregation sign suggested the existence of significant cauda equina injuries.

Keywords Paraplegia, Thoracolumbar fracture, Magnetic resonance imaging, Electrodiagnosis, Prognosis licenses/by-nc/4.0) which permits unrestricted noncommercial use, distribution, and reproduction in any medium, provided the original work is properly cited. Copyright $\odot 2015$ by Korean Academy of Rehabilitation Medicine 


\section{INTRODUCTION}

The thoracolumbar junction is susceptible to injury and is the most commonly injured portion of the spine [1-3]. This junction is a highly vulnerable axial region because of biomechanical stresses and its location; the distal spinal cord or epiconus, conus medullaris, and cauda equina are proximal [4]. Following blunt trauma and injury of the thoracolumbar junction, $10 \%$ of injuries were at $\mathrm{T} 12$, $16 \%$ were at $\mathrm{L} 1$, and $15 \%$ were at the $\mathrm{L} 2$ vertebral level [5]. Conus medullaris syndrome (CMS) or cauda equina syndrome (CES) generally occurs following injury to this portion of the spine.

CMS is caused by injury to the lower end of the spinal cord (conus) and lumbar nerve roots. A detailed anatomical review in adult humans found that the conus medullaris had a variable location, and is found between the T12 and L2 vertebral levels [6]. CMS is characterized by a combination of upper and lower motor neuron signs including saddle anesthesia, areflexic bladder and bowel, and varying degrees of lower extremity weakness. Most cases of CMS result from spinal trauma including fractures [4].

CES refers to an injury of the lumbosacral nerve roots within the spinal canal. This condition is considered a pure lower motor neuron injury with the absence of upper motor neuron signs, and it is characterized by asym- metric lower extremity weakness. Similar to CMS, CES presents with symptoms consistent with saddle anesthesia, bladder and bowel dysfunction, and variable lower extremity involvement. It occurs following severe lower lumbar disc herniations, spinal stenosis, traumas, tumors, and post-surgical epidural hematomas [7].

Neurological recovery from injury to the conus medullaris or cauda equina is unpredictable. They are difficult to differentiate from each other on the basis of clinical outcomes or electrodiagnostic features. Magnetic resonance imaging (MRI) allows improved visualization of the spinal cord parenchyma and adjacent soft tissue.

The nerve root sedimentation sign has recently been described as a means to discriminate between patients with and without symptomatic lumbar spinal stenosis (LSS) [8-13]. The lumbar nerve roots in patients without LSS sink to the dorsal part of the dural sac, as seen on a supine MRI, as a result of gravity (Fig. 1A). A positive sedimentation sign was defined as the absence of nerve root sedimentation, with a large number of nerve roots located in the central part of the dural sac [8-13] (Fig. 1B). A previous study showed that patients with LSS have increased epidural pressure at the level of the stenosis; the absence of sedimentation of the lumbar nerve roots may be caused by a tethering of the affected nerve roots [13]. As in patients with LSS, we hypothesized that the nerve root sedimentation sign would be positive in paraplegics
(A)
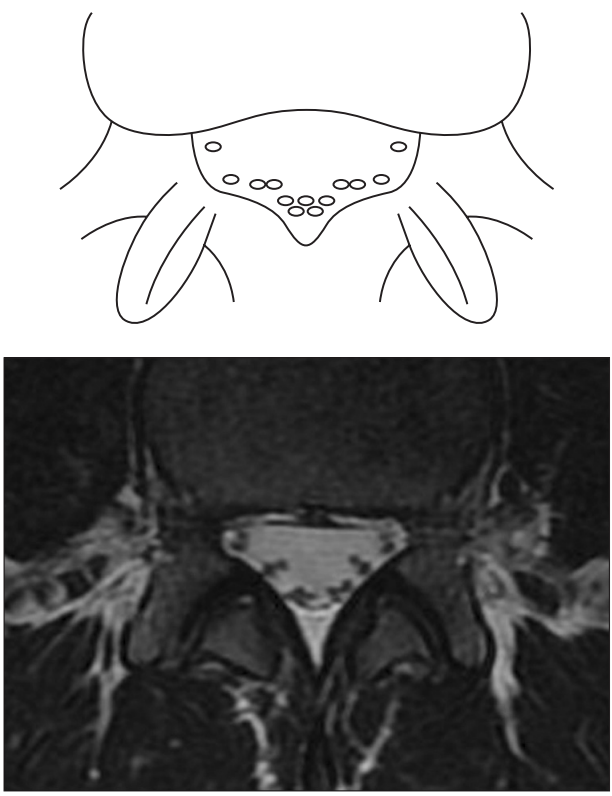

(B)
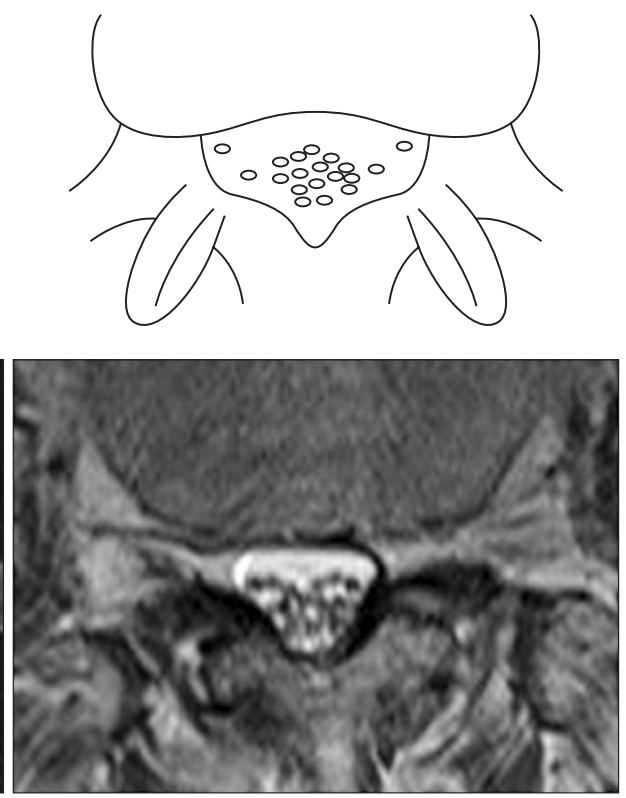

Fig. 1. Schematic illustration of the nerve root sedimentation sign in comparison with magnetic resonance imaging scans. (A) Negative sedimentation sign showed that lumbar nerve roots sink to the dorsal part of the dural sac as a result of gravity. (B) Positive sedimentation sign was defined as the absence of nerve root sedimentation. 

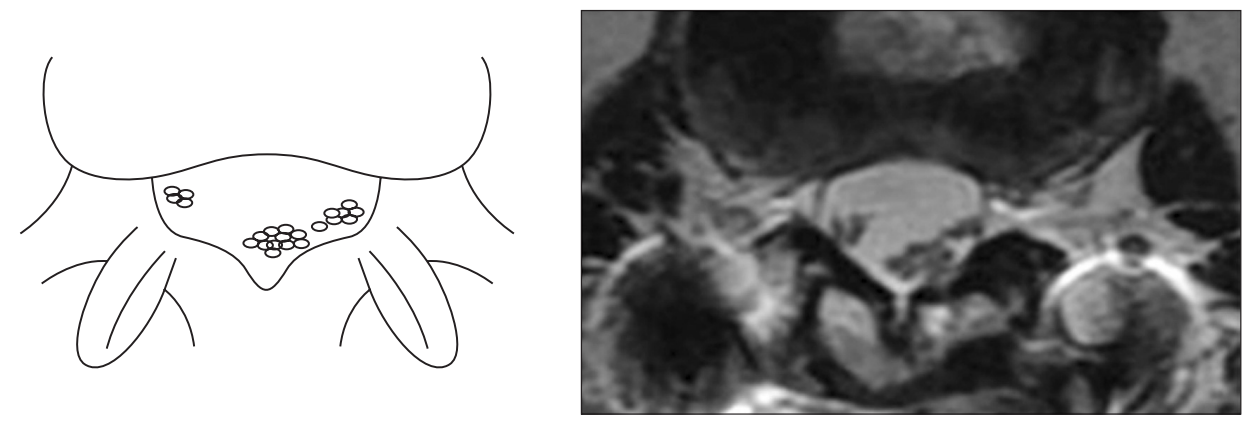

Fig. 2. Schematic illustration of the root aggregation sign in comparison with magnetic resonance imaging scans. The root aggregation sign was defined as the presence of root aggregation.

with thoracolumbar fracture because of neural insult or possibly altered epidural pressure caused by mechanical impact.

In axial MRI scans, an additional phenomenon can be observed. In paraplegics with thoracolumbar fracture, aggregation of nerve roots is sometimes seen. We termed this the root aggregation sign. A positive root aggregation sign was defined as the presence of root aggregation as evidenced-on at least one axial MRI scan (Fig. 2). We also hypothesized that the root aggregation sign suggested the presence of a root lesion in cases of conus medullaris syndrome or cauda equina syndrome.

High signal intensity on T2-weighted MRI scans in cervical spondylotic myelopathy was reported; patients with increased signal intensity had poor clinical results after either conservative or surgical treatment [14]. Signal intensity changes in patients with cervical spondylotic myelopathy have been studied as high signal intensity is thought to reflect pathological changes in the spinal cord and has a prognostic value [15-19]. In this study we assessed the signal intensity changes in cases of conus medullaris.

To the best of our knowledge, there has been no study about predicting neurological recovery in paraplegics with thoracolumbar fracture using MRI findings. Therefore, the objective of this study was to investigate the usefulness of MRI findings for predicting neurological recovery in paraplegics with thoracolumbar fracture using association analysis with clinical outcomes and electrodiagnostic features.

\section{MATERIAL AND METHODS}

\section{Subjects}

Clinical outcomes, electrodiagnostic features, and MRI findings of persons with paraplegia after thoracolumbar fracture were retrospectively reviewed. One hundred eighteen patients who were identified had a history of thoracic or lumbar fracture, and in whose cases electrodiagnostic and MRI data was available. All had visited the department of rehabilitation medicine at a tertiary medical center from August 2005 to March 2014. Patients who had a history of thoracolumbar fracture (level of lesion, T12-L2) and neurologic paraplegia were included. Patients with lower extremity weakness caused by other pre-existing diseases, such as myopathy, local nerve entrapments, and peripheral nerve lesions, were excluded. Of the 118 patients, 30 (19 males and 11 females) met the inclusion criteria. The study was reviewed and approved by the Institutional Review Board.

\section{Methods}

\section{Clinical outcomes}

Neurological examinations of all patients were performed according to the guidelines of the American Spinal Injury Association (ASIA) which assess motor and sensory functions. Neurological deficit was assessed by the ASIA impairment scale (AIS). To prevent misdiagnosis in the early stages of acute paraplegia caused by spinal shock or recovery from transient neurological deficits, the final clinical classification was noted. The outcome of ambulatory capacity of each patient was assessed and classified in one of four categories: 1) full ambulatory capacity, where there is an independent gait; 2) functional ambulatory capacity, where daily walking was possible over short distances using a cane; 3) therapeutic ambulatory capacity, where standing and walking were possible but only with a walker; and 4) no ambulatory capacity, where the patient is unable to walk or stand. This categorization was consistent with that used in earlier studies in which the outcome of ambulatory capacity was used to assess and predict clinical outcomes in spinal cord injury 
(A)
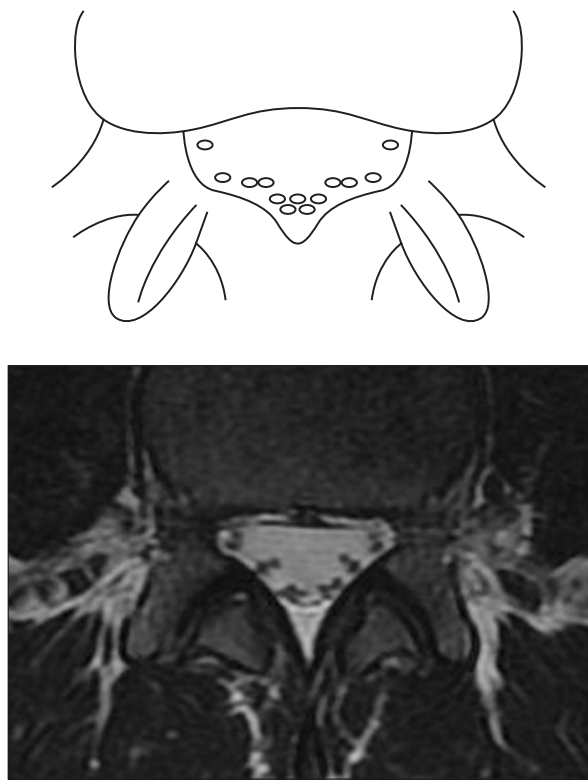

(B)
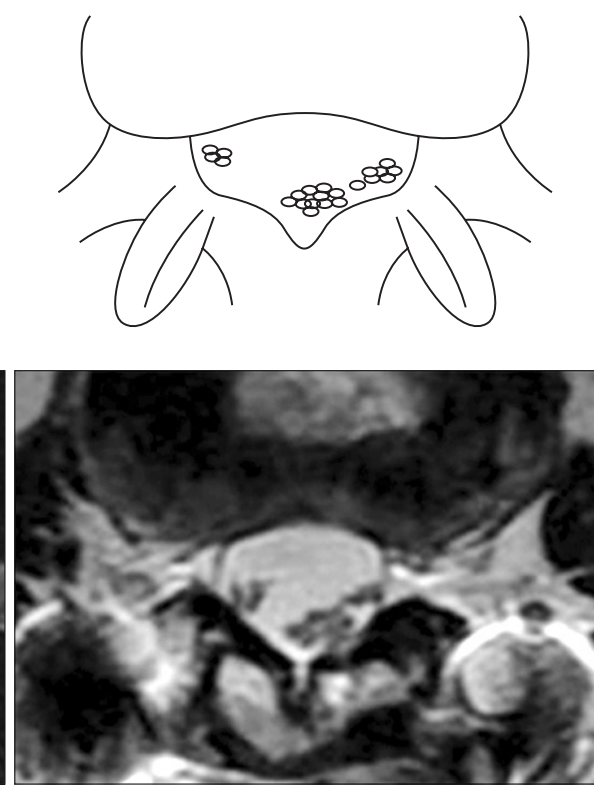

(C)
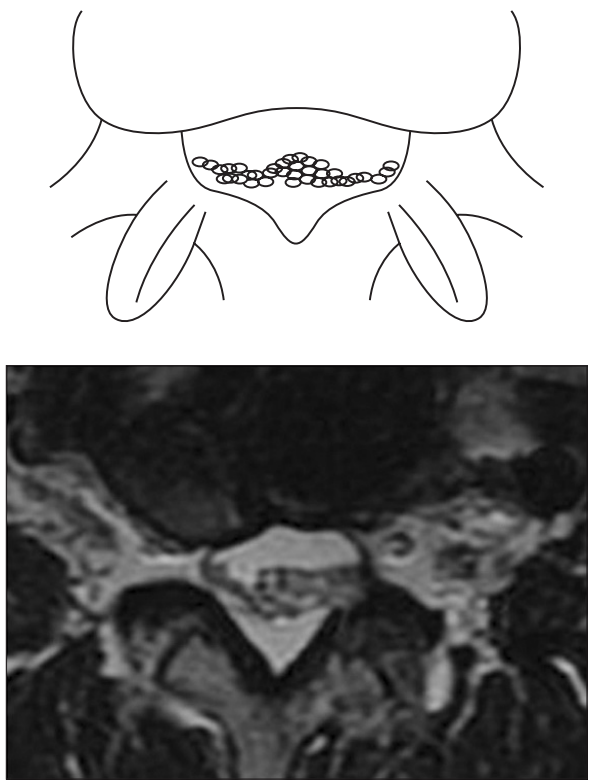

Fig. 3. Grading of the root aggregation sign in comparison with magnetic resonance imaging scans depending on the amount of nerve root aggregation. (A) Grade 0, no aggregation; (B) Grade 1, partial aggregation; (C) Grade 2, total aggregation.

[20-22]. These clinical outcomes were assessed at baseline (at the time of admission to the department of rehabilitation medicine) and at follow-up (after 2-3 months of a rehabilitation program). Recovery of ambulatory capacity was defined as having better outcome of ambulatory capacity at follow-up than at baseline.

\section{Electrodiagnostic features}

Motor nerve potentials were recorded using surface electrodes placed over the extensor digitorum brevis and the abductor hallucis to assess the common peroneal and tibial nerves, respectively. Other nerve conduction studies included both tibial H-reflexes and sural sensory and superficial peroneal sensory recordings. Motor-evoked potential (MEP) was measured in the abductor hallucis following transcranial stimulation of the motor cortex. The average time of electrodiagnostic evaluation after onset of paraplegia was $7.9 \pm 14.3$ months.

The degree of lower limb compound motor action potential (CMAP) was assessed and assigned to one of four categories: 1) normal in both the common peroneal and tibial nerves; 2) reduced amplitude in either the common peroneal or tibial nerve; 3 ) reduced amplitude in both the common peroneal and tibial nerves or no response in either the common peroneal or tibial nerve; or 4) no response in both the common peroneal and tibial nerves. The degree of H-reflex and the degree of MEP was also assessed and assigned to one of three categories: 1) normal in both tibial nerves; 2 ) delayed latency in either one or both tibial nerves; or 3) no response in either one or both tibial nerves. This classification was chosen because it is thought to reflect different degrees of both common peroneal and tibial nerve CMAP amplitude and impaired impulse transmission in the spinal canal.

\section{MRI findings}

Each participant underwent lumbar spinal MRI including axial and sagittal scans with a 1.5 Tesla MRI unit (General Electric, Milwaukee, WI, USA). Slices were 4 $\mathrm{mm}$ wide, and the acquisition matrix was $512 \times 256$. Using these images, the nerve root sedimentation sign, the root aggregation sign, and the signal intensity changes in the conus medullaris were independently assessed by two raters who had no knowledge of clinical history or diagnosis. The average time between onset of paraplegia and MRI evaluation was $8.6 \pm 20.5$ months.

Using the methods described by a previous study, the nerve root sedimentation sign was measured in axial 
MRI scans [8-13]. Positive and negative sedimentation signs distinguished the absence or presence of nerve root sedimentation in the dorsal part of the dural sac, respectively (Fig. 1A, 1B). The root aggregation sign indicated the presence of root aggregation in at least one axial MRI scan (Fig. 2) and was assigned a grade from 0 to 3 depending on the amount of nerve root aggregation (grade 0 , no aggregation; grade 1 , partial aggregation; grade 2 , total aggregation) (Fig. 3). Signal intensity changes were defined by focal high-signal intensity of conus medullaris on T2-weighted MRI scans.

In the current study, each axial MRI scan obtained at the mid-vertebral level from L1 to L5 including one level below where the fracture is present. Levels above the fracture were excluded as the nerve root sedimentation

Table 1. Patient demographics

\begin{tabular}{|rr}
\hline \multicolumn{1}{c}{ Characteristic } & \multicolumn{1}{c}{ Value } \\
\hline Age (yr) & $62.3 \pm 15.8$ \\
Sex & $19(63.3)$ \\
\hline Male & $11(36.7)$ \\
\hline Female & \\
\hline Fracture level & $5(14.3)$ \\
\hline T12 & $16(45.7)$ \\
\hline L1 & $14(40.0)$ \\
\hline L2 & $4(13.3)$ \\
\hline Type of injury & $26(86.7)$ \\
\hline Traffic accident & \\
\hline Slips or falls & $7.9 \pm 14.3$ \\
\hline Mean duration from onset (mo) & $8.6 \pm 20.5$ \\
\hline Electrodiagnostic evaluation & \\
\hline MRI evaluation &
\end{tabular}

Values are presented as mean \pm standard deviation or number (\%).

MRI, magnetic resonance imaging. and the root aggregation signs are not present above the T12 level.

\section{Statistical analyses}

Pearson chi-square test was used to examine the association between clinical outcomes and MRI findings, and between electrodiagnostic features and MRI findings. Spearman rank correlation coefficient was used to assess the relationship between the grade of the root aggregation sign and electrodiagnostic features. Cohen's kappa was used to examine inter-rater reliability. SPSS ver. 17.0 (SPSS, Chicago, IL, USA) statistical software was used for all analyses. A p-value $<0.05$ was considered statistically significant.

\section{RESULTS}

The 30 patients (19 males, 11 females) were $62.3 \pm 15.8$ years of age. Their basic clinical characteristics are shown in Table 1. Injuries were resultant of traffic accidents $(n=4$, $13.3 \%)$ and slips or falls $(n=26,86.7 \%)$. Fractures in most subjects $(85.7 \%)$ were at levels $\mathrm{L} 1$ and $\mathrm{L} 2$; the remaining fractures were at the T12 vertebral level (14.3\%). Interrater reliability is presented in Table 2 . Inter-rater reliability for the nerve root sedimentation sign and the root aggregation sign was $\kappa=0.67$ and $\kappa=0.78$, respectively.

Table 2. Inter-rater reliability

\begin{tabular}{|c|c|c|c|}
\hline $\begin{array}{c}\text { MRI } \\
\text { findings }\end{array}$ & Raters & $\begin{array}{c}\text { Inter-rater } \\
\text { reliability }(\kappa)\end{array}$ & p-value \\
\hline SedSign & Rater 1 vs. Rater 2 & 0.67 & $0.001^{*}$ \\
\hline AggSign & Rater 1 vs. Rater 2 & 0.78 & $<0.001^{*}$ \\
\hline
\end{tabular}

MRI, magnetic resonance imaging; SedSign, the nerve root sedimentation sign; AggSign, the root aggregation sign. ${ }^{*} \mathrm{p}<0.05$

Table 3. Relationship of clinical outcomes to MRI findings

\begin{tabular}{lccr}
\hline & CM signal intensity changes & \multicolumn{1}{c}{ SedSign } & \multicolumn{1}{c}{ AggSign } \\
\hline AIS & $1.147(\mathrm{p}=0.284)$ & $1.841(\mathrm{p}=0.175)$ & $0.719(\mathrm{p}=0.397)$ \\
Outcome of ambulatory capacity at baseline & $1.990(\mathrm{p}=0.158)$ & $3.671(\mathrm{p}=0.055)$ & $0.410(\mathrm{p}=0.840)$ \\
Outcome of ambulatory capacity at follow-up & $1.718(\mathrm{p}=0.190)$ & $0.057(\mathrm{p}=0.812)$ & $<0.001(\mathrm{p}=1.000)$ \\
Recovery of ambulatory capacity & $0.818(\mathrm{p}=0.366)$ & $4.854\left(\mathrm{p}=0.028^{*}\right)$ & $0.818(\mathrm{p}=0.366)$ \\
\hline
\end{tabular}

MRI, magnetic resonance imaging; AIS, American Spinal Injury Association impairment scale; CM, conus medullaris; SedSign, the nerve root sedimentation sign; AggSign, the root aggregation sign.

${ }^{*} \mathrm{p}<0.05$ by Pearson chi-square test. 
Table 4. Relationship of electrodiagnostic features to MRI findings

\begin{tabular}{lccc} 
& CM signal intensity change & SedSign & AggSign \\
\hline Degree of lower limb CMAP & $0.877(\mathrm{p}=0.349)$ & $0.000(\mathrm{p}=0.987)$ & $5.026\left(\mathrm{p}=0.025^{*}\right)$ \\
Degree of H-reflex & $0.253(\mathrm{p}=0.615)$ & $1.612(\mathrm{p}=0.204)$ & $0.405(\mathrm{p}=0.524)$ \\
Degree of MEP & $1.611(\mathrm{p}=0.204)$ & $0.607(\mathrm{p}=0.436)$ & $0.039(\mathrm{p}=0.844)$ \\
\hline
\end{tabular}

MRI, magnetic resonance imaging; CMAP, compound motor action potential; MEP, motor evoked potential; CM, conus medullaris; SedSign, the nerve root sedimentation sign; AggSign, the root aggregation sign.

${ }^{*} \mathrm{p}<0.05$ by Pearson chi-square test.

Table 5. Relationship of the grade of the root aggregation sign to electrodiagnostic features by the Spearman correlation coefficient

\begin{tabular}{lc}
\hline & AggSign grade $^{\mathrm{a})}$ \\
\hline Degree of lower limb CMAP & $0.488\left(\mathrm{p}=0.008^{*}\right)$ \\
Degree of H-reflex & $0.240(\mathrm{p}=0.238)$ \\
Degree of MEP & $0.240(\mathrm{p}=0.238)$ \\
\hline
\end{tabular}

CMAP, compound motor action potential; MEP, motor evoked potential; AggSign, the root aggregation sign.

${ }^{a)}$ AggSign grade: negative, partial and total.

${ }^{*} \mathrm{p}<0.05$.

\section{Association between clinical outcomes and MRI findings}

Concerning the relationship of clinical outcomes to MRI findings, recovery of ambulatory capacity was associated with a positive nerve root sedimentation sign (Table 3). Neurological deficits assessed by the AIS and the outcome of ambulatory capacity of patients measured at both baseline and follow-up were not associated with any MRI findings. In patients who showed some improvement of ambulatory capacity at the follow-up evaluation, the nerve root sedimentation sign on MRI scans was shown to have a close association with this outcome.

\section{Association between electrodiagnostic features and} MRI findings

Significant associations were evident between the degree of lower limb CMAP and the root aggregation sign in the relationship between electrodiagnostic features and MRI findings (Table 4). The root aggregation sign was associated with reduced CMAP amplitude in the common peroneal and tibial nerves. The degree of lower limb CMAP was also positively correlated with the grade of the root aggregation sign (Table 5). The maximum nerve root sedimentation sign and root aggregation sign was $70.6 \%$ and $81.8 \%$, respectively, in the mid-vertebral region one level below the fracture site. Signal intensity changes in conus medullaris did not have significant association with clinical outcomes or electrodiagnostic features (Tables 3, 4).

\section{DISCUSSION}

The spinal cord usually ends at the L1-2 vertebral level in adults and continues as the filum terminale and cauda equina. The thoracolumbar region, where the rigid thoracic kyphosis transitions into the mobile lumbar lordosis, is particularly susceptible to traumatic injuries [23]. Adjacent to this junction, the transition from central to peripheral nervous system occurs with associated nerve dilation. Patients with an injury above the conus medullaris typically present with symptoms consistent with spinal cord injury, whereas those with injuries below this level present with symptoms consistent with lumbosacral radiculopathies. Traumatic lesions of the cauda equina, which cause acute neurological deterioration as opposed to the gradual onset of lower motor neuron dysfunction, generally have a poorer prognosis [24].

If neurological insults occur in the upper lumbar spinal region including the thoracolumbar junction, lesions of the motor neurons and nerve roots can occur. It is difficult to differentiate lesions of motor neurons from those of nerve roots by clinical outcomes or electrodiagnostic features in acute paraplegic patients. This is because spinal responses in lower limb reflexes in acute paraplegia are diminished and muscle tone is reduced in both conus medullaris and cauda equina lesions. However, the differentiation between the two conditions is important in the prognosis and treatment of patients. In conus medullaris lesions, the patient will develop a combination of upper and lower motor neuron signs. In contrast, a cauda equina lesion is associated with lower motor neuron deficits in the absence of upper motor neuron signs. 
In a previous study of the correlation with clinical outcomes, electrodiagnostic features, and MRI findings, CMAPs of the median and ulnar nerves in tetraplegic patients had diagnostic and prognostic value in assessing the extent of damage to motor nerve fibers and predicting the outcome of hand function [25]. Another study showed that in symptomatic lumbar stenosis patents, lower limb electrodiagnostic features did not correlate with MRI findings, although H-reflex and F-wave testing correlated with the location of stenosis [26].

MRI is commonly used to evaluate the spinal cord and nerve roots in lumbar spine disorders. Because the extent of nerve damage is an important clinical indicator, the relationship between MRI findings and nerve damage related to clinical outcomes is important.

The purpose of this study was to analyze MRI findings of paraplegics with thoracolumbar fracture to differentiate lesions of the motor neuron from those of the nerve roots and to evaluate the usefulness of MRI findings for predicting neurological recovery using association analysis with clinical outcomes and electrodiagnostic features. There were three main findings. First, the nerve root sedimentation sign was significantly associated with recovery of ambulatory capacity. Second, the root aggregation sign was associated with reduced CMAP amplitude in common peroneal and tibial nerves. Third, both of these signs were obtained mainly one level below the midvertebral fracture site.

A positive sedimentation sign was defined as an absence of nerve root sedimentation and was quite useful in discriminating between patients with and without LSS [8-13]. A retrospective review of MRI scans showed that this sign is most accurate in defining severe LSS cases [8]. Another study reported that an absence of nerve root sedimentation is related to arachnoiditis, possibly caused by degenerative spinal diseases including LSS $[27,28]$. In the acute inflammatory stage, production of a fibrinous exudate causes thickening of the nerve roots and perineum, and causes them to stick to each other and to the thecal sac, producing central clumping [27].

A positive sedimentation sign was significantly associated with improvement of ambulatory capacity in paraplegics with thoracolumbar fracture. This sign had no association with any other electrodiagnostic features or clinical outcomes. We expected that this sign would allow for differentiation between patients with nerve root injury from those with spinal cord injury. However, the findings suggest that a positive sedimentation sign on its own cannot differentiate root injury from spinal cord injury. Rather, this sign was associated with recovery of ambulatory capacity in follow-up measurements and not indicative of persistent paralysis.

The root aggregation sign was defined as the presence of nerve root aggregation within the spinal canal. In axial MRI scans, we frequently observed this sign in patients with paraplegia with thoracolumbar fracture. A positive aggregation sign was associated with reduced CMAP amplitude of the common peroneal and tibial nerves. This sign suggested the existence of significant cauda equina injuries. We also demonstrated a significant correlation between grade of the root aggregation sign and the degree of lower limb CMAP, implicating this new MRI grading system provides a useful prognostic tool (Fig. 3).

This study has several limitations. As a retrospective analysis of previously obtained data, there were variations in the number of cases examined by each the clinicians who performed the physical examinations. In addition, the study was limited by the relatively small sample size and heterogeneity of the study group. A prospective study with a large sample size would be helpful for analyzing association factors and increasing statistical power. Another limitation is the lack of other functional measurements such as the functional independence measure or the spinal cord independence measure. These measurements apply to paraplegics with thoracolumbar fractures like spinal cord injuries, but in this study patients were not routinely measured with these rating systems.

In conclusion, several MRI findings were significantly associated with clinical outcomes and electrodiagnostic features. The nerve root sedimentation sign was significantly associated with recovery of ambulatory capacity. The root aggregation sign was associated with reduced CMAP amplitude of the common peroneal and tibial nerves in paraplegics with thoracolumbar fracture.

\section{CONFLICT OF INTEREST}

No potential conflict of interest relevant to this article was reported. 


\section{REFERENCES}

1. Prabhakar MM, Rao BS, Patel L. Thoracolumbar burst fracture with complete paraplegia: rationale for second-stage anterior decompression and fusion regarding functional outcome. J Orthop Traumatol 2009;10: 83-90.

2. Mikles MR, Stchur RP, Graziano GP. Posterior instrumentation for thoracolumbar fractures. J Am Acad Orthop Surg 2004;12:424-35.

3. Kirkpatrick AW, McKevitt E. Thoracolumbar spine fractures: is there a problem? Can J Surg 2002;45:21-4.

4. Kingwell SP, Curt A, Dvorak MF. Factors affecting neurological outcome in traumatic conus medullaris and cauda equina injuries. Neurosurg Focus 2008;25:E7.

5. Holmes JF, Miller PQ, Panacek EA, Lin S, Horne NS, Mower WR. Epidemiology of thoracolumbar spine injury in blunt trauma. Acad Emerg Med 2001;8:866-72.

6. Malas MA, Salbacak A, Buyukmumcu M, Seker M, Koyluoglu B, Karabulut AK. An investigation of the conus medullaris termination level during the period of fetal development to adulthood. Kaibogaku Zasshi 2001;76:453-9.

7. Gardner A, Gardner E, Morley T. Cauda equina syndrome: a review of the current clinical and medicolegal position. Eur Spine J 2011;20:690-7.

8. Tomkins-Lane CC, Quint DJ, Gabriel S, Melloh M, Haig AJ. Nerve root sedimentation sign for the diagnosis of lumbar spinal stenosis: reliability, sensitivity, and specificity. Spine (Phila Pa 1976) 2013;38:E155460.

9. Macedo LG, Wang Y, Battie MC. The sedimentation sign for differential diagnosis of lumbar spinal stenosis. Spine (Phila Pa 1976) 2013;38:827-31.

10. Staub LP, Barz T, Melloh M, Lord SJ, Chatfield M, Bossuyt PM. Clinical validation study to measure the performance of the Nerve Root Sedimentation Sign for the diagnosis of lumbar spinal stenosis. Contemp Clin Trials 2011;32:470-4.

11. Barz T, Staub LP, Melloh M, Hamann G, Lord SJ, Chatfield MD, et al. Clinical validity of the nerve root sedimentation sign in patients with suspected lumbar spinal stenosis. Spine J 2014;14:667-74.

12. Barz T, Melloh M, Staub LP, Lord SJ, Lange J, Roder CP, et al. Nerve root sedimentation sign: evaluation of a new radiological sign in lumbar spinal stenosis. Spine
(Phila Pa 1976) 2010;35:892-7.

13. Barz T, Melloh M, Staub LP, Lord SJ, Lange J, Merk HR. Increased intraoperative epidural pressure in lumbar spinal stenosis patients with a positive nerve root sedimentation sign. Eur Spine J 2014;23:985-90.

14. Takahashi M, Sakamoto Y, Miyawaki M, Bussaka H. Increased MR signal intensity secondary to chronic cervical cord compression. Neuroradiology 1987;29: 550-6.

15. Mehalic TF, Pezzuti RT, Applebaum BI. Magnetic resonance imaging and cervical spondylotic myelopathy. Neurosurgery 1990;26:217-27.

16. Matsuda Y, Miyazaki K, Tada K, Yasuda A, Nakayama T, Murakami $\mathrm{H}$, et al. Increased MR signal intensity due to cervical myelopathy: analysis of 29 surgical cases. J Neurosurg 1991;74:887-92.

17. Al-Mefty O, Harkey LH, Middleton TH, Smith RR, Fox JL. Myelopathic cervical spondylotic lesions demonstrated by magnetic resonance imaging. J Neurosurg 1988;68:217-22.

18. Okada Y, Ikata T, Yamada H, Sakamoto R, Katoh S. Magnetic resonance imaging study on the results of surgery for cervical compression myelopathy. Spine (Phila Pa 1976) 1993;18:2024-9.

19. Wada E, Yonenobu K, Suzuki S, Kanazawa A, Ochi T. Can intramedullary signal change on magnetic resonance imaging predict surgical outcome in cervical spondylotic myelopathy? Spine (Phila Pa 1976) 1999;24:455-62.

20. Curt A, Dietz V. Ambulatory capacity in spinal cord injury: significance of somatosensory evoked potentials and ASIA protocol in predicting outcome. Arch Phys Med Rehabil 1997;78:39-43.

21. Crozier KS, Cheng LL, Graziani V, Zorn G, Herbison G, Ditunno JF Jr. Spinal cord injury: prognosis for ambulation based on quadriceps recovery. Paraplegia 1992;30:762-7.

22. Hussey RW, Stauffer ES. Spinal cord injury: requirements for ambulation. Arch Phys Med Rehabil 1973;54:544-7.

23. Court-Brown CM, McQueen MM, Tornetta P. Trauma. Philadelphia: Lippincott Williams \& Wilkins; 2006. p. 226-7.

24. Kostuik JP, Harrington I, Alexander D, Rand W, Evans D. Cauda equina syndrome and lumbar disc herniation. J Bone Joint Surg Am 1986;68:386-91. 
25. Curt A, Dietz V. Nerve conduction study in cervical spinal cord injury: significance for hand function. NeuroRehabilitation 1996;7:165-73.

26. Chiodo A, Haig AJ, Yamakawa KS, Quint D, Tong H, Choksi VR. Magnetic resonance imaging vs. electrodiagnostic root compromise in lumbar spinal stenosis: a masked controlled study. Am J Phys Med Rehabil 2008;87:789-97.
27. Jackson A, Isherwood I. Does degenerative disease of the lumbar spine cause arachnoiditis? A magnetic resonance study and review of the literature. $\mathrm{Br} \mathrm{J} \mathrm{Ra-}$ diol 1994;67:840-7.

28. Laitt R, Jackson A, Isherwood I. Patterns of chronic adhesive arachnoiditis following Myodil myelography: the significance of spinal canal stenosis and previous surgery. Br J Radiol 1996;69:693-8. 